\title{
A Research on a Certain Family of Numbers and Polynomials Related to Stirling Numbers, Central Factorial Numbers, and Euler Numbers
}

\author{
J. Y. Kang and C. S. Ryoo \\ Department of Mathematics, Hannam University, Daejeon 306-791, Republic of Korea \\ Correspondence should be addressed to J. Y. Kang; rkdwjddnr2002@yahoo.co.kr
}

Received 28 June 2013; Accepted 23 August 2013

Academic Editor: Ch. Tsitouras

Copyright (C) 2013 J. Y. Kang and C. S. Ryoo. This is an open access article distributed under the Creative Commons Attribution License, which permits unrestricted use, distribution, and reproduction in any medium, provided the original work is properly cited.

Recently, many mathematicians have studied different kinds of the Euler, Bernoulli, and Genocchi numbers and polynomials. In this paper, we give another definition of polynomials $\widetilde{U}_{n}(x)$. We observe an interesting phenomenon of "scattering" of the zeros of the polynomials $\widetilde{U}_{n}(x)$ in complex plane. We find out some identities and properties related to polynomials $\widetilde{U}_{n}(x)$. Finally, we also derive interesting relations between polynomials $\widetilde{U}_{n}(x)$, Stirling numbers, central factorial numbers, and Euler numbers.

\section{Introduction}

Recently, many mathematicians have studied in the areas of the Bernoulli numbers and polynomials, Euler numbers and polynomials, Genocchi numbers and polynomials, Stirling numbers, and central factorial numbers (see [1-17]). These numbers and polynomials possess many interesting properties and are arising in many areas of mathematics and physics. In this paper, we give another definition of polynomials $\widetilde{U}_{n}(x)$. We obtain some interesting identities and properties related to polynomials $\widetilde{U}_{n}(x)$. In order to study the polynomials $\widetilde{U}_{n}(x)$, we must understand the structure of the polynomials $\widetilde{U}_{n}(x)$. Therefore, using computer, a realistic study for the polynomials $\widetilde{U}_{n}(x)$ is very interesting. It is the aim of this paper to observe an interesting phenomenon of "scattering" of the zeros of the polynomials $\widetilde{U}_{n}(x)$ in complex plane.

The Stirling numbers of the first kind $s(n, k)$ define that

$$
\sum_{k=0}^{n} s(n, k) x^{k}=x(x-1)(x-2) \cdots(x-n+1) .
$$

The generating function of (1) is as follows:

$$
(\log (1+x))^{k}=k ! \sum_{n=k}^{\infty} s(n, k) \frac{x^{n}}{n !} .
$$

From (1) and (2), we are aware of some properties of the Stirling numbers of the first kind $s(n, k)$ as follows (see $[1,2,15]$ ):

$$
s(n, k)=s(n-1, k-1)-(n-1) s(n-1, k),
$$

with

$$
\begin{gathered}
s(n, 0)=0 \quad(n \in \mathbb{N}), \\
s(n, n)=1 \quad\left(n \in \mathbb{Z}^{+}=\mathbb{N} \cup 0\right), \\
s(n, 1)=(-1)^{n-1}(n-1) ! \quad(n \in \mathbb{N}), \\
s(n, k)=0 \quad(k>n \text { or } k<0) .
\end{gathered}
$$

We usually define the central factorial numbers $T(n, k)$ as the following expansion formula (see $[1,7])$ :

$$
\sum_{k=0}^{n} T(n, k) x\left(x-1^{2}\right)\left(x-2^{2}\right) \cdots\left(x-(k-1)^{2}\right)=x^{n} .
$$

The generating function of (5) is as follows:

$$
\left(e^{x}+e^{-x}-2\right)^{k}=(2 k) ! \sum_{n=k}^{\infty} T(n, k) \frac{x^{2 n}}{(2 n) !} .
$$


By using (5) and (6), we are aware of some properties of the central factorial numbers $T(n, k)$ as follows:

$$
\begin{gathered}
T(0,0)=1, \\
T(n, 0)=0 \quad(n \in \mathbb{N}), \\
T(n, 1)=1 \quad(n \in \mathbb{N}), \\
T(n, k)=T(n-1, k-1)+k^{2} T(n-1, k),
\end{gathered}
$$

with

$$
\begin{gathered}
T(n, 2)=\frac{1}{4}\left(4^{n-1}-1\right), \\
T(n, 3)=\frac{9^{n}}{360}-\frac{4^{n}}{60}+\frac{1}{24} \quad(n \in \mathbb{N}) .
\end{gathered}
$$

The Euler numbers $E_{n}$ are defined by the generating function:

$$
\frac{2}{e^{t}+1}=\sum_{n=0}^{\infty} E_{n} \frac{t^{n}}{n !}
$$

We introduce the Euler polynomials $E_{n}(x)$ as follows:

$$
\left(\frac{2}{e^{t}+1}\right) e^{x t}=\sum_{n=0}^{\infty} E_{n}(x) \frac{t^{n}}{n !} .
$$

Zhi-Hong Sun introduces the sequence $\left\{U_{n}\right\}$ similar to Euler numbers as follows:

$$
U_{0}=1, \quad U_{n}=-2 \sum_{k=1}^{[n / 2]}\left(\begin{array}{c}
n \\
2 k
\end{array}\right) U_{n-2 k} \quad(n \geq 1),
$$

where $[x]$ is the greatest integer not exceeding $x$ (see $[4,5]$ ).

The outline of this paper is as follows. In Section 2, this paper is to define polynomials $\widetilde{U}_{n}(x)$. In Section 3 , we describe the beautiful zeros of the polynomials $\widetilde{U}_{n}(x)$ using a numerical investigation. We investigate the roots of the polynomials $\widetilde{U}_{n}(x)$. Also we carried out computer experiments for demonstrating a remarkably regular structure of the complex roots of the polynomials $\widetilde{U}_{n}(x)$. In Section 4 , we derive some special relations of polynomials $\widetilde{U}_{n}(x)$ and Euler numbers.

\section{Some Properties Involving a Certain Family of Numbers and Polynomials}

In this section, we introduce the polynomials $\widetilde{U}_{n}(x)$ and investigate some interesting properties and identities which are related to polynomials $\widetilde{U}_{n}(x)$. We also try to find relations between polynomials $\widetilde{U}_{n}(x)$, Stirling numbers $s(n, k)$, and central factorial numbers $T(n, k)$.

Definition 1. For $x \in \mathbb{R}$ or $\mathbb{C}$, the polynomials $\widetilde{U}_{n}(x)$ are defined by

$$
\sum_{n=0}^{\infty} \widetilde{U}_{n}(x) \frac{t^{n}}{n !}=\left(\frac{1}{e^{t}+e^{-t}-1}\right)^{x} e^{x t}
$$

From Definition 1, we have Theorem 2.
Theorem 2. Let $n, k, l \in \mathbb{Z}^{+}$and $n \geq k-l-1$. Then one obtains

$$
\begin{aligned}
\widetilde{U}_{2 n}(x)= & \sum_{k=0}^{2 n} \sum_{l=0}^{[k / 2]}\left(\begin{array}{c}
2 n \\
2 l
\end{array}\right)(-1)^{k-2 l} \\
& \times \sum_{j=k-2 l}^{n-l} \frac{(2 j) !}{j !} T(n-l, j) s(j, k-2 l) x^{k}, \\
\widetilde{U}_{2 n+1}(x)= & \sum_{k=1}^{2 n+1} \sum_{l=0}^{k[k-1 / 2]}\left(\begin{array}{l}
2 n+1 \\
2 l-1
\end{array}\right)(-1)^{k-(2 l+1)} \\
& \times \sum_{j=k-(2 l+1)}^{n-l} \frac{(2 j) !}{j !} T(n-l, j) s(j, k-(2 l+1)) x^{k},
\end{aligned}
$$

where $[x]$ is the greatest integer not exceeding $x$ and $\mathbb{Z}^{+}=\mathbb{N} U$ $\{0\}$.

Proof. By Definition 1, (2), and (6), we have

$$
\begin{aligned}
\sum_{n=0}^{\infty} \widetilde{U}_{n}(x) \frac{t^{n}}{n !} & \left(\frac{1}{e^{t}+e^{-t}-1}\right)^{x} e^{t x} \\
& =\sum_{j=0}^{\infty}\left(\begin{array}{c}
x+j-1 \\
j
\end{array}\right)(-1)^{j}\left(e^{t}+e^{-t}-2\right)^{j} e^{t x} \\
& =\sum_{j=0}^{\infty}(-1)^{j}\left(\begin{array}{c}
x+j-1 \\
j
\end{array}\right)(2 j) ! \sum_{n=j}^{\infty} T(n, j) \frac{t^{2 n}}{(2 n) !} e^{t x} \\
& =\sum_{n=0}^{\infty} \sum_{j=0}^{n}(-1)^{j}\left(\begin{array}{c}
x+j-1 \\
j
\end{array}\right)(2 j) ! T(n, j) \frac{t^{2 n}}{(2 n) !} e^{t x} \\
& =\sum_{n=0}^{\infty} \sum_{j=0}^{n} \sum_{k=0}^{j}(-1)^{k} s(j, k) x^{k} \frac{(2 j) !}{j !} T(n, j) \frac{t^{2 n}}{(2 n) !} \sum_{n=0}^{\infty} x^{n} \frac{t^{n}}{n !} .
\end{aligned}
$$

From now on, we have to consider odd terms and even terms by using Cauchy product. So, we get to generating terms by dividing the odd terms and the even terms, respectively,

$$
\sum_{n=0}^{\infty} \widetilde{U}_{n}(x) \frac{t^{n}}{n !}=\sum_{n=0}^{\infty} \widetilde{U}_{2 n}(x) \frac{t^{2 n}}{(2 n) !}+\sum_{n=0}^{\infty} \widetilde{U}_{2 n+1}(x) \frac{t^{2 n+1}}{(2 n+1) !} .
$$

The following equation is the generating even terms:

$$
\begin{aligned}
& \widetilde{U}_{2 n}(x) \\
& =\sum_{l=0}^{n}\left(\begin{array}{c}
2 n \\
2 l
\end{array}\right) \sum_{j=0}^{n-l} \frac{(2 j) !}{j !} T(n-l, j) \sum_{k=0}^{j}(-1)^{k} s(j, k) x^{k+2 l} \\
& =\sum_{k=0}^{2 n} \sum_{l=0}^{[k / 2]}\left(\begin{array}{c}
2 n \\
2 l
\end{array}\right)(-1)^{k-2 l} \sum_{j=k-2 l}^{n-l} \frac{(2 j) !}{j !} T(n-l, j) s(j, k-2 l) x^{k},
\end{aligned}
$$

where $[x]$ is the greatest integer not exceeding $x$. 
We also derive the generating odd terms:

$$
\begin{aligned}
& \widetilde{U}_{2 n+1}(x) \\
& =\sum_{l=0}^{n}\left(\begin{array}{c}
2 n+1 \\
2 l+1
\end{array}\right) \sum_{j=0}^{n-l} \frac{(2 j) !}{j !} T(n-l, j) \sum_{k=0}^{j}(-1)^{k} s(j, k) x^{k+(2 l+1)}
\end{aligned}
$$

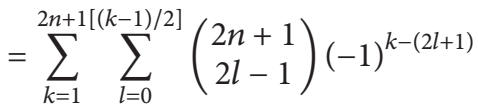

$$
\begin{aligned}
& \times \sum_{j=k-(2 l+1)}^{n-l} \frac{(2 j) !}{j !} T(n-l, j) s(j, k-(2 l+1)) x^{k},
\end{aligned}
$$

where $[x]$ is the greatest integer not exceeding $x$.

Thus, we complete the proof of Theorem 2.

Example 3. Let $n=1,2,3,4,5,6,7,8$. Then we can know the following polynomials:

$$
\begin{aligned}
& \widetilde{U}_{1}(x)=x, \\
& \widetilde{U}_{2}(x)=x^{2}-2 x, \\
& \widetilde{U}_{3}(x)=x^{3}-6 x^{2}, \\
& \widetilde{U}_{4}(x)=x^{4}-12 x^{3}+12 x^{2}+10 x, \\
& \widetilde{U}_{5}(x)=x^{5}-20 x^{4}+60 x^{3}+50 x^{2}, \\
& \widetilde{U}_{6}(x)=x^{6}-30 x^{5}+180 x^{4}+30 x^{3}-300 x^{2}-182 x, \\
& \widetilde{U}_{7}(x)=x^{7}-42 x^{6}+420 x^{5}-490 x^{4}-2100 x^{3}-1274 x^{2}, \\
& \widetilde{U}_{8}(x)=x^{8}-56 x^{7}+840 x^{6}-2660 x^{5}-6720 x^{4} \\
& \\
& +
\end{aligned}
$$

Corollary 4. Let $n, l \in \mathbb{Z}^{+}$and $n \geq l$. Then one has

$$
\begin{aligned}
\widetilde{U}_{2 n}(x)= & \sum_{l=0}^{n}\left(\begin{array}{c}
2 n \\
2 l
\end{array}\right) \sum_{j=0}^{n-l} \frac{(2 j) !}{j !} T(n-l, j) \sum_{k=0}^{j}(-1)^{k} s(j, k) x^{k+2 l}, \\
\widetilde{U}_{2 n+1}(x)= & \sum_{l=0}^{n}\left(\begin{array}{c}
2 n+1 \\
2 l+1
\end{array}\right) \sum_{j=0}^{n-l} \frac{(2 j) !}{j !} T(n-l, j) \\
& \times \sum_{k=0}^{j}(-1)^{k} s(j, k) x^{k+(2 l+1)} .
\end{aligned}
$$

Proof. Corollary 4 shows identities of $\widetilde{U}_{2 n}(x)$ and $\widetilde{U}_{2 n+1}(x)$. The proof of Corollary 4 is contained in Theorem 2.

Setting $x=1$ in Corollary 4 , we easily see the following corollary.
Corollary 5. Relation of $\widetilde{U}_{n}(1)$ and central factorial numbers is shown.

Let $n, l, j \in \mathbb{Z}^{+}$and $n \geq l+j$. Then one has

$$
\begin{aligned}
\widetilde{U}_{2 n}(1) & =\sum_{l=0}^{n}\left(\begin{array}{c}
2 n \\
2 l
\end{array}\right) \sum_{j=0}^{n-l}(-1)^{j}(2 j) ! T(n-l, j), \\
\widetilde{U}_{2 n+1}(1) & =\sum_{l=0}^{n}\left(\begin{array}{c}
2 n+1 \\
2 l+1
\end{array}\right) \sum_{j=0}^{n-l}(-1)^{j}(2 j) ! T(n-l, j) .
\end{aligned}
$$

Proof. Consider the following:

$$
\begin{aligned}
& \widetilde{U}_{2 n}(x) \\
& =\sum_{l=0}^{n}\left(\begin{array}{c}
2 n \\
2 l
\end{array}\right) \sum_{j=0}^{n-l} \frac{(2 j) !}{j !} T(n-l, j) \sum_{k=0}^{j}(-1)^{k} s(j, k) x^{k+2 l} \\
& =\sum_{l=0}^{n}\left(\begin{array}{c}
2 n \\
2 l
\end{array}\right) \sum_{j=0}^{n-l} \frac{(2 j) !}{j !} T(n-l, j)(-1)^{2 j} \sum_{k=0}^{j}(-1)^{k} s(j, k) x^{k+2 l} \\
& =\sum_{l=0}^{n}\left(\begin{array}{c}
2 n \\
2 l
\end{array}\right) \sum_{j=0}^{n-l} \frac{(2 j) !}{j !}(-1)^{-j} T(n-l, j) \sum_{k=0}^{j}(-1)^{k-j} s(j, k) x^{k+2 l} .
\end{aligned}
$$

We find out the following equation from the above equation by substituting $x=1$ :

$$
\begin{aligned}
\widetilde{U}_{2 n}(1) & =\sum_{l=0}^{n}\left(\begin{array}{c}
2 n \\
2 l
\end{array}\right) \sum_{j=0}^{n-l} \frac{(2 j) !}{j !} T(n-l, j) j ! \\
& =\sum_{l=0}^{n}\left(\begin{array}{c}
2 n \\
2 l
\end{array}\right) \sum_{j=0}^{n-l}(-1)^{j}(2 j) ! T(n-l, j) .
\end{aligned}
$$

We also can find out $\widetilde{U}_{2 n+1}(1)$ by using similar method as above:

$$
\begin{aligned}
\widetilde{U}_{2 n+1}(x)= & \sum_{l=0}^{n}\left(\begin{array}{c}
2 n+1 \\
2 l+1
\end{array}\right) \sum_{j=0}^{n-l} \frac{(2 j) !}{j !} T(n-l, j) \\
& \times \sum_{k=0}^{j}(-1)^{k} s(j, k) x^{k+2 l} \\
= & \sum_{l=0}^{n}\left(\begin{array}{c}
2 n+1 \\
2 l+1
\end{array}\right) \sum_{j=0}^{n-l} \frac{(2 j) !}{j !} T(n-l, j)(-1)^{2 j} \\
& \times \sum_{k=0}^{j}(-1)^{k} s(j, k) x^{k+2 l} \\
= & \sum_{l=0}^{n}\left(\begin{array}{c}
2 n+1 \\
2 l+1
\end{array}\right) \sum_{j=0}^{n-l} \frac{(2 j) !}{j !}(-1)^{-j} T(n-l, j) \\
& \times \sum_{k=0}^{j}(-1)^{k-j} s(j, k) x^{k+2 l} .
\end{aligned}
$$


If we substitute $x=1$, then

$$
\begin{aligned}
\widetilde{U}_{2 n+1}(1) & =\sum_{l=0}^{n}\left(\begin{array}{c}
2 n+1 \\
2 l+1
\end{array}\right) \sum_{j=0}^{n-l} \frac{(2 j) !}{j !} T(n-l, j) j ! \\
& =\sum_{l=0}^{n}\left(\begin{array}{c}
2 n+1 \\
2 l+1
\end{array}\right) \sum_{j=0}^{n-l}(-1)^{j}(2 j) ! T(n-l, j) .
\end{aligned}
$$

Let $n, k, l \in \mathbb{Z}^{+}$and $n \geq k-l-1$ and

$$
\begin{aligned}
a(2 n, k)= & \sum_{l=0}^{[k / 2]}\left(\begin{array}{c}
2 n \\
2 l
\end{array}\right)(-1)^{k-2 l} \\
& \times \sum_{j=k-2 l}^{n-l} \frac{(2 j) !}{j !} T(n-l, j) s(j, k-2 l), \\
b(2 n+1, k)= & \sum_{l=0}^{[(k-1) / 2]}\left(\begin{array}{c}
2 n+1 \\
2 l-1
\end{array}\right)(-1)^{k-(2 l+1)} \\
& \times \sum_{j=k-(2 l+1)}^{n-l} \frac{(2 j) !}{j !} T(n-l, j) s(j, k-(2 l+1)),
\end{aligned}
$$

where $[x]$ is the greatest integer not exceeding $x$.

Then we have

$$
\widetilde{U}_{2 n}(x)=\sum_{k=0}^{2 n} a(2 n, k) x^{k}, \quad \widetilde{U}_{2 n+1}(x)=\sum_{k=1}^{2 n+1} b(2 n+1, k) x^{k} .
$$

By using $a(2 n, k)$ and $b(2 n+1, k)$, we obtain Theorem 6 .

Theorem 6. Let $n, k \in \mathbb{Z}^{+}$. Then one gets

$$
\begin{gathered}
\widetilde{U}_{2 n}(1)= \begin{cases}1 & \text { if } n=0 \\
2 \sum_{k=0}^{n-1} a(2 n, 2 k)-2^{2 n}+1 & \text { if } n \geq k+1,\end{cases} \\
\widetilde{U}_{2 n+1}(1)=2 \sum_{k=1}^{n} b(2 n+1,2 k)+2^{2 n+1}-1 . \\
\widetilde{U}_{2 n}(1)= \begin{cases}1 & \text { if } n=0 \\
2 \sum_{k=0}^{n-1} a(2 n, 2 k+1)+2^{n}-1 & \text { if } n \geq k+1,\end{cases} \\
\widetilde{U}_{2 n+1}(1)=2 \sum_{k=1}^{2 n+1} b(2 n+1,2 k-1)-2^{2 n+1}+1 .
\end{gathered}
$$

Proof. From Definition 1, one easily obtains the following equation:

$$
\begin{aligned}
\sum_{n=0}^{\infty} \widetilde{U}_{n}(x) \frac{t^{n}}{n !} & =\sum_{n=0}^{\infty} \widetilde{U}_{2 n}(x) \frac{t^{2 n}}{(2 n) !}+\sum_{n=0}^{\infty} \widetilde{U}_{2 n+1}(x) \frac{t^{2 n+1}}{(2 n+1) !} \\
& =\left(\frac{e^{t}}{e^{t}+e^{-t}-1}\right)^{x} .
\end{aligned}
$$

We also easily get the following equation by substituting $x=$ -1 :

$$
\begin{aligned}
\sum_{n=0}^{\infty} \widetilde{U}_{n}(-1) \frac{t^{n}}{n !}= & \sum_{n=0}^{\infty} \widetilde{U}_{2 n}(-1) \frac{t^{2 n}}{(2 n) !} \\
& +\sum_{n=0}^{\infty} \widetilde{U}_{2 n+1}(-1) \frac{t^{2 n+1}}{(2 n+1) !} \\
= & \left(\frac{e^{t}}{e^{t}+e^{-t}-1}\right)^{-1} \\
= & 1+\sum_{n=0}^{\infty}(-1)^{n}\left(2^{n}-1\right) \frac{t^{n}}{n !} \\
= & 1+\sum_{n=0}^{\infty}(-1)^{n}\left(2^{n}-1\right) \frac{t^{n}}{n !} \\
= & 1+\sum_{n=1}^{\infty}\left(2^{2 n}-1\right) \frac{t^{2 n}}{2 n !} \\
& -\sum_{n=0}^{\infty}\left(2^{2 n+1}-1\right) \frac{t^{2 n+1}}{(2 n+1) !} .
\end{aligned}
$$

Thus,

$$
\begin{aligned}
\sum_{n=0}^{\infty} \widetilde{U}_{2 n}(-1) \frac{t^{2 n}}{(2 n) !} & =1+\sum_{n=1}^{\infty}\left(2^{2 n}-1\right) \frac{t^{2 n}}{2 n !} \\
\widetilde{U}_{2 n+1}(-1) & =1-2^{2 n+1} .
\end{aligned}
$$

We have to consider odd terms and even terms in (26) and the previous equations, respectively.

Odd terms are in the following form:

$$
\begin{aligned}
\widetilde{U}_{2 n+1}(-1) & =\sum_{k=1}^{2 n+1} b(2 n+1, k)(-1)^{k} \\
& =\sum_{k=1}^{n} b(2 n+1,2 k)-\sum_{k=1}^{n+1} b(2 n+1,2 k-1) \\
& =1-2^{2 n+1}, \\
\widetilde{U}_{2 n+1}(1) & =2 \sum_{k=1}^{n} b(2 n+1,2 k)+2^{2 n+1}-1 \\
& =2 \sum_{k=1}^{2 n+1} b(2 n+1,2 k-1)-2^{2 n+1}+1,
\end{aligned}
$$

and even terms are similar to the way of the proof process of the odd terms. Therefore, we omit the proof process of the even terms.

Theorem 7. Let $n, k \in \mathbb{Z}^{+}$and $k+1 \leq n$. Then one has

$$
\widetilde{U}_{n+1}(1)-\widetilde{U}_{n}(1)=\sum_{k=0}^{n-1}\left(\begin{array}{l}
n \\
k
\end{array}\right)\left(\left(1-2^{n-k}\right) \widetilde{U}_{k+1}(1)-\widetilde{U}_{k}(1)\right) \text {. }
$$


Proof. From Definition 1, one easily obtains the following equation:

$$
\sum_{n=0}^{\infty} \widetilde{U}_{n}(1) \frac{t^{n}}{n !}=\left(\frac{e^{t}}{e^{t}+e^{-t}-1}\right)
$$

We also easily get the following by using differential, namely,

$$
\sum_{n=1}^{\infty} \widetilde{U}_{n}(1) \frac{t^{n-1}}{(n-1) !}=\frac{-e^{t}+2}{\left(e^{t}+e^{-t}-1\right)^{2}} .
$$

Thus,

$$
\begin{aligned}
\left(e^{2 t}-e^{t}+1\right) \sum_{n=1}^{\infty} \widetilde{U}_{n}(1) \frac{t^{n-1}}{(n-1) !} \\
=\left(e^{2 t}-e^{t}+1\right) \frac{-e^{t}+2}{\left(e^{t}+e^{-t}-1\right)^{2}} \\
=\left(2-e^{t}\right) \sum_{n=0}^{\infty} \widetilde{U}_{n}(1) \frac{t^{n}}{n !}
\end{aligned}
$$

Then, right-hand side is in the following form:

$$
\begin{aligned}
\left(2-e^{t}\right) & \sum_{n=0}^{\infty} \widetilde{U}_{n}(1) \frac{t^{n}}{n !} \\
= & 2 \sum_{n=0}^{\infty} \widetilde{U}_{n}(1) \frac{t^{n}}{n !}-\sum_{n=0}^{\infty} \sum_{k=0}^{\infty}\left(\begin{array}{l}
n \\
k
\end{array}\right) \widetilde{U}_{k}(1) \frac{t^{n}}{n !} \\
= & \sum_{n=0}^{\infty}\left(2 \widetilde{U}_{n}(1)-\sum_{k=0}^{n}\left(\begin{array}{l}
n \\
k
\end{array}\right) \widetilde{U}_{k}(1)\right) \frac{t^{n}}{n !},
\end{aligned}
$$

and left-hand side is in the following form:

$$
\begin{aligned}
& \left(e^{2 t}-e^{t}+1\right) \sum_{n=1}^{\infty} \widetilde{U}_{n}(1) \frac{t^{n-1}}{(n-1) !} \\
& =\sum_{n=0}^{\infty} 2^{n} \frac{t^{n}}{n !} \sum_{n=0}^{\infty} \widetilde{U}_{n+1}(1) \frac{t^{n}}{n !}-\sum_{n !}^{\infty} \sum_{n=0}^{\infty} \widetilde{U}_{n+1}(1) \frac{t^{n}}{n !}+\sum_{n=0}^{\infty} \widetilde{U}_{n+1}(1) \frac{t^{n}}{n !} \\
& =\sum_{n=0}^{\infty}\left(\sum_{k=0}^{n}\left(\begin{array}{l}
n \\
k
\end{array}\right)\left(2^{n-k}-1\right) \widetilde{U}_{k+1}(1)+\widetilde{U}_{n+1}(1)\right) \frac{t^{n}}{n !} .
\end{aligned}
$$

By using comparing coefficients of $t^{n} / n !$ in the previous equations, we can represent the equation; that is,

$$
\begin{gathered}
\sum_{k=0}^{n}\left(\begin{array}{l}
n \\
k
\end{array}\right)\left(2^{n-k}-1\right) \widetilde{U}_{k+1}(1)+\widetilde{U}_{n+1}(1) \\
=2 \widetilde{U}_{n}(1)-\sum_{k=0}^{n}\left(\begin{array}{l}
n \\
k
\end{array}\right) \widetilde{U}_{k}(1) .
\end{gathered}
$$

By simple calculation, we get

$$
\begin{aligned}
\widetilde{U}_{n+1} & (1)-2 \widetilde{U}_{n}(1) \\
= & \sum_{k=0}^{n}\left(\begin{array}{l}
n \\
k
\end{array}\right)\left(1-2^{n-k}\right) \widetilde{U}_{k+1}(1)-\sum_{k=0}^{n}\left(\begin{array}{l}
n \\
k
\end{array}\right) \widetilde{U}_{k}(1) \\
= & \sum_{k=0}^{n-1}\left(\begin{array}{l}
n \\
k
\end{array}\right)\left(\left(1-2^{n-k}\right) \widetilde{U}_{k+1}(1)-\widetilde{U}_{k}(1)\right)-\widetilde{U}_{n}(1) .
\end{aligned}
$$

Therefore, we proved that

$$
\widetilde{U}_{n+1}(1)-\widetilde{U}_{n}(1)=\sum_{k=0}^{n-1}\left(\begin{array}{l}
n \\
k
\end{array}\right)\left(\left(1-2^{n-k}\right) \widetilde{U}_{k+1}(1)-\widetilde{U}_{k}(1)\right) .
$$

\section{Zeros of the Polynomials $\widetilde{U}_{n}(x)$}

In this section, we investigate the reflection symmetry of the zeros of the polynomials $\widetilde{U}_{n}(x)$.

We investigate the beautiful zeros of the polynomials $\widetilde{U}_{n}(x)$ by using a computer. We plot the zeros of the polynomials $\widetilde{U}_{n}(x)$ for $n=20,30,40,50$ and $x \in \mathbb{C}$ (Figure 1). In Figure 1(a), we choose $n=20$. In Figure 1(b), we choose $n=30$. In Figure 1(c), we choose $n=40$. In Figure 1(d), we choose $n=50$.

Throughout the numerical experiments, we can finally conclude that polynomials $\widetilde{U}_{n}(x)$ have no $\operatorname{Re}(x)=0$ reflection symmetry analytic complex functions. However, we observe that $\widetilde{U}_{n}(x)$ has $\operatorname{Im}(x)=0$ reflection symmetry (see Figures 1,2, and 3). The obvious corollary is that the zeros of $\widetilde{U}_{n}(x)$ will also inherit these symmetries:

$$
\text { if } \widetilde{U}_{n}(x)=0, \quad \text { then } \widetilde{U}_{n}\left(x^{*}\right)=0,
$$

where $*$ denotes complex conjugation (see Figure 1 ).

Plots of real zeros of $\widetilde{U}_{n}(x)$ for $1 \leq n \leq 50$ structure are presented (Figure 2).

Our numerical results for approximate solutions of real zeros of the $\widetilde{U}_{n}(x)$ are displayed (Tables 1 and 2).

We observe a remarkably regular structure of the complex roots of the polynomials $\widetilde{U}_{n}(x)$. We hope to verify a remarkably regular structure of the complex roots of the polynomials $\widetilde{U}_{n}(x)$ (Table 1).

Stacks of zeros of $\widetilde{U}_{n}(x)$ for $1 \leq n \leq 50$ from a 3-D structure are presented (Figure 3).

Next, we calculated an approximate solution satisfying $\widetilde{U}_{n}(x), x \in \mathbb{R}$. The results are given in Table 2 .

Finally, we will consider the more general problems. Find the numbers of complex zeros $C_{\widetilde{U}_{n}(x)}$ of $\widetilde{U}_{n}(x), \operatorname{Im}(x) \neq 0$. Since $n$ is the degree of the polynomial $\widetilde{U}_{n}(x)$, the number of real zeros $R_{\widetilde{U}_{n}(x)}$ lying on the real plane $\operatorname{Im}(x)=0$ is then 


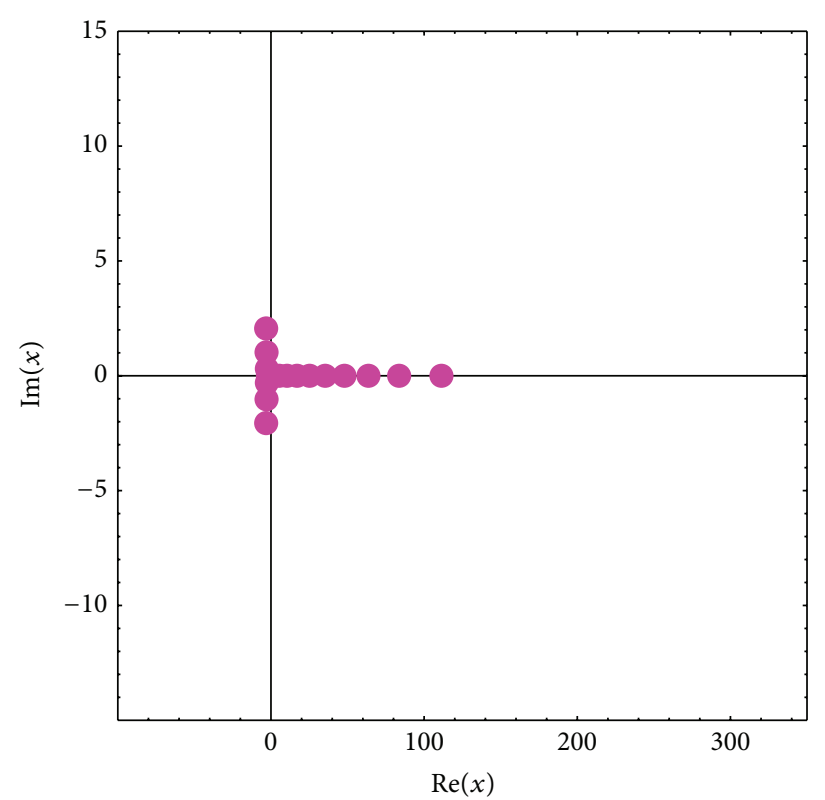

(a)

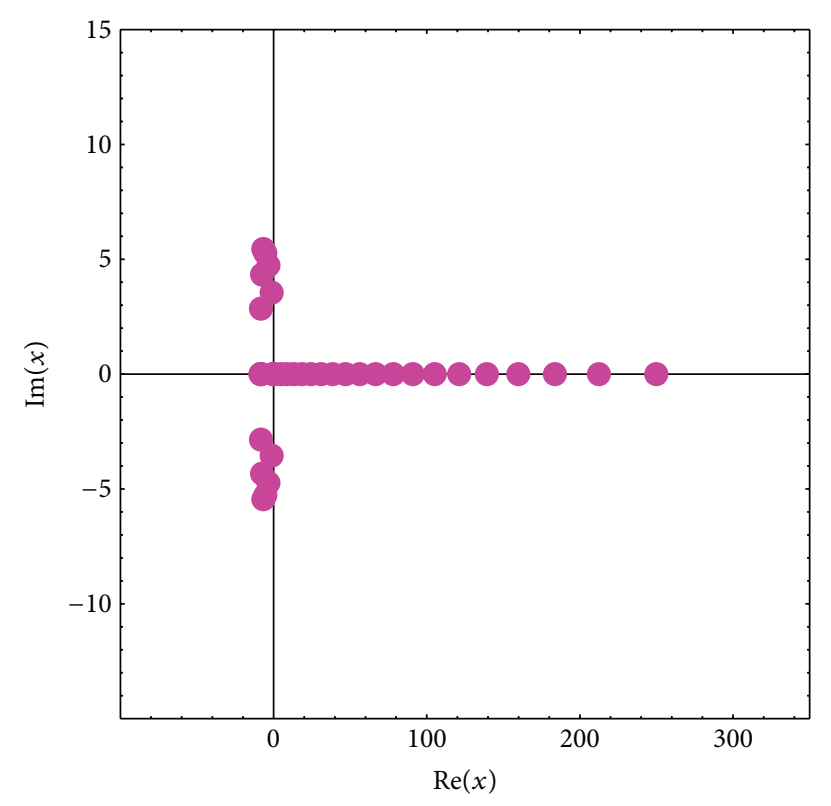

(c)

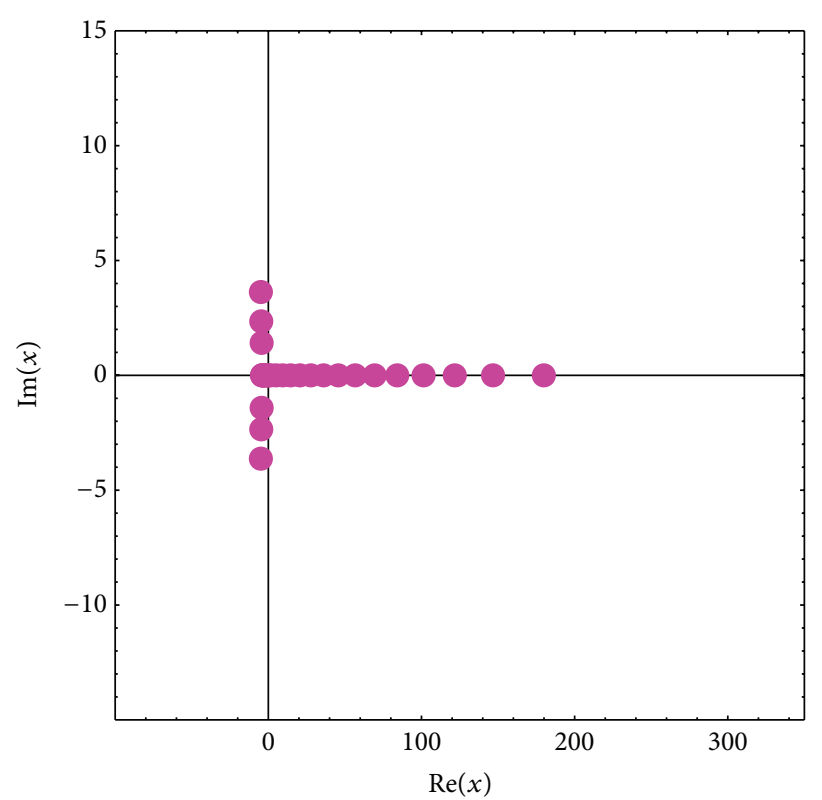

(b)

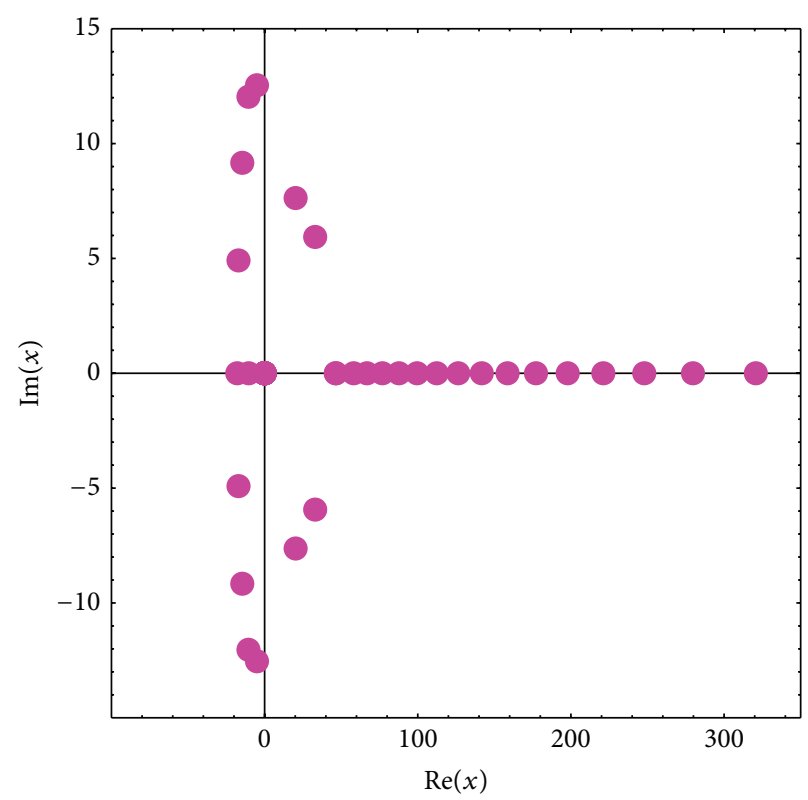

(d)

Figure 1: Zeros of $\widetilde{U}_{n}(x)$ for $n=20,30,40,50$.

$R_{\widetilde{U}_{n}(x)}=n-C_{\widetilde{U}_{n}(x)}$, where $C_{\widetilde{U}_{n}(x)}$ denotes complex zeros. See Table 1 for tabulated values of $R_{\widetilde{U}_{n}(x)}$ and $C_{\widetilde{U}_{n}(x)}$. We plot the zeros of $\widetilde{U}_{n}(x)$, respectively (Figures 1-3). These figures give mathematicians an unbounded capacity to create visual mathematical investigations of the behavior of the roots of the $\widetilde{U}_{n}(x)$. Moreover, it is possible to create new mathematical ideas and analyze them in ways that generally are not possible by hand. The authors have no doubt that investigation along this line will lead to a new approach employing numerical methods in the field of research of $\widetilde{U}_{n}(x)$ to appear in mathematics and physics.

\section{Some Relations of Polynomials $\widetilde{U}_{n}(x)$ and Euler Numbers}

In this section, we find out interesting relations between polynomials $\widetilde{U}_{n}(x)$ and Euler numbers. We usually use the values of polynomials $\widetilde{U}_{n}(x)$ and Euler polynomials. 


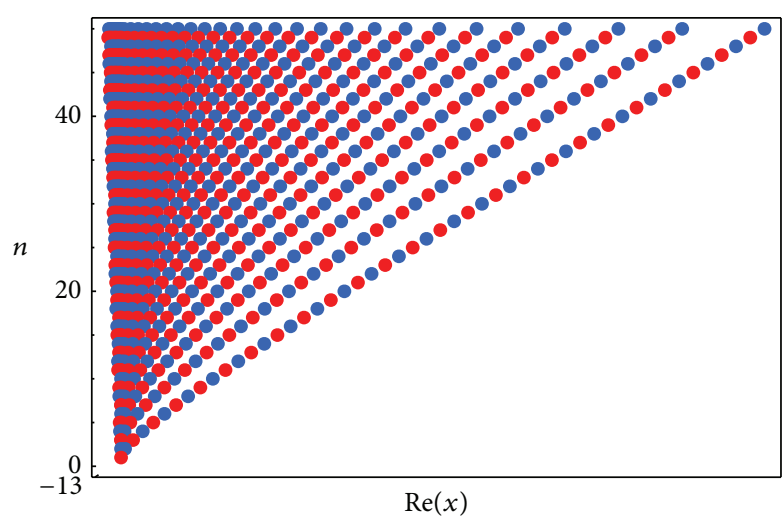

FIGURE 2: Real zeros of $\widetilde{U}_{n}(x)$ for $1 \leq n \leq 50$.

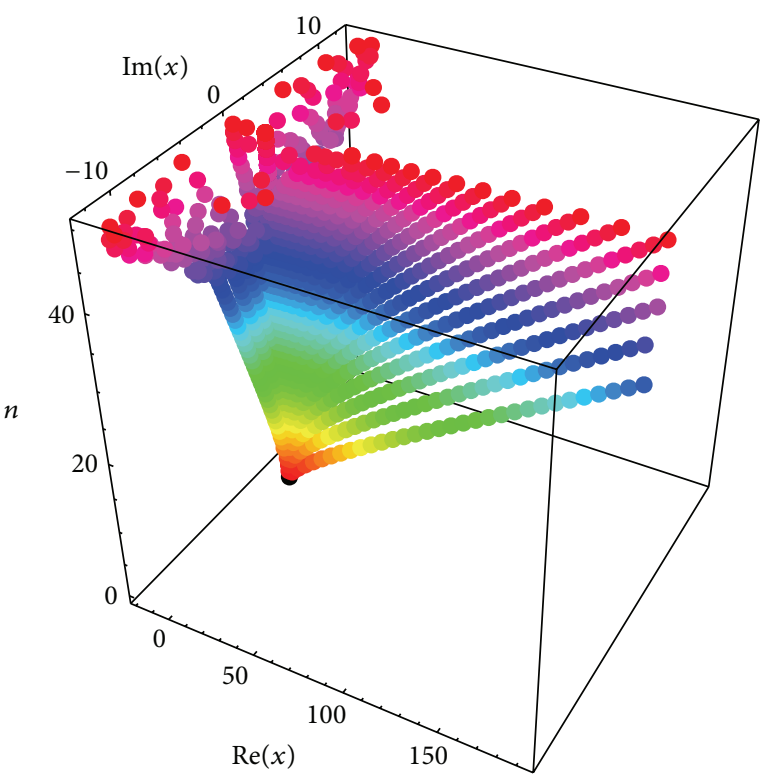

FIGURE 3: Stacks of zeros of $\widetilde{U}_{n}(x)$ for $1 \leq n \leq 50$.

TABLE 1: Numbers of real and complex zeros of $\widetilde{U}_{n}(x)$.

\begin{tabular}{lcc}
\hline Degree $n$ & Real zeros & Complex zeros \\
\hline 1 & 1 & 0 \\
2 & 2 & 0 \\
3 & 3 & 0 \\
4 & 4 & 0 \\
5 & 5 & 0 \\
6 & 4 & 2 \\
7 & 5 & 2 \\
8 & 6 & 2 \\
9 & 5 & 4 \\
10 & 6 & 4 \\
$\vdots$ & $\vdots$ & $\vdots$ \\
30 & 20 & 10 \\
\hline
\end{tabular}

TABLE 2: Approximate solutions of $\widetilde{U}_{n}(x)=x \in \mathbb{R}$.

\begin{tabular}{lc}
\hline$n$ & $x$ \\
\hline 1 & 0 \\
2 & 0,2 \\
3 & $-0.5346,0,0,1.73111,10.8035$ \\
4 & $-0.6759,0,0,4.60224,16.0736$ \\
5 & $0,1.64591338,8.2083255,21.6537433$ \\
6 & $0,0,4.0761207,12.3245041,27.4570253$ \\
7 & $-0.877412,0,1.6058053,7.1033690,16.8165987,33.4298423$ \\
8 & $-0.968751,0,0,3.8044567,10.5908618,21.598401,39.536481$ \\
9 & $0,1.5827926,6.4946365,14.442719,26.611139,45.751858$ \\
\hline
\end{tabular}

Theorem 8. Let $n \in \mathbb{Z}^{+}$. Then one has

$$
\begin{aligned}
& \frac{2}{3^{n}} \widetilde{U}_{n}(1)=E_{n}\left(\frac{2}{3}\right)+(-1)^{n} E_{n}, \\
& \frac{2}{3^{n}} \widetilde{U}_{n}(2)=\left(E\left(\frac{2}{3}\right)-E\right)^{n}+2^{n-1}\left(E_{n}\left(\frac{2}{3}\right)+(-1)^{n} E_{n}\right),
\end{aligned}
$$

with the usual convention of replacing $E^{n}$ by $E_{n}$.

Proof. By setting $x=1$ in Definition 1 and using Euler numbers, then we have

$$
\begin{aligned}
\sum_{n=0}^{\infty} \widetilde{U}_{n}(1) \frac{t^{n}}{n !} & =\left(\frac{e^{t}}{e^{t}+e^{-t}-1}\right) \\
& =\frac{1}{1+e^{-3 t}}+\frac{1}{1+e^{3 t}} e^{2 t} \\
& =\frac{1}{2} \sum_{n=0}^{\infty} 3^{n}\left((-1)^{n} E_{n}+E_{n}\left(\frac{2}{3}\right)\right) \frac{t^{n}}{n !} .
\end{aligned}
$$

Hence,

$$
\frac{2}{3^{n}} \widetilde{U}_{n}(1)=E_{n}\left(\frac{2}{3}\right)+(-1)^{n} E_{n} .
$$

We also obtain the above equation by taking $x=2$ and using Euler numbers:

$$
\begin{aligned}
\sum_{n=0}^{\infty} \widetilde{U}_{n}(2) \frac{t^{n}}{n !}= & \left(\frac{e^{t}}{e^{t}+e^{-t}-1}\right)^{2} \\
= & \left(\frac{1}{1+e^{-3 t}}+\frac{1}{1+e^{3 t}} e^{2 t}\right) \\
& \times\left(\frac{1}{1+e^{-3 t}}+\frac{1}{1+e^{3 t}} e^{2 t}\right) \\
= & \left(\frac{1}{2} \sum_{n=0}^{\infty} 3^{n}\left((-1)^{n} E_{n}+E_{n}\left(\frac{2}{3}\right)\right) \frac{t^{n}}{n !}\right) \\
& \times\left(\frac{1}{2} \sum_{n=0}^{\infty} 3^{n}\left((-1)^{n} E_{n}+E_{n}\left(\frac{2}{3}\right)\right) \frac{t^{n}}{n !}\right) .
\end{aligned}
$$


By using Cauchy product in the above equation, we have

$$
\begin{aligned}
\sum_{n=0}^{\infty} \widetilde{U}_{n}(2) \frac{t^{n}}{n !}= & \frac{1}{4} \sum_{n=0}^{\infty} \sum_{k=0}^{n}\left(\begin{array}{l}
n \\
k
\end{array}\right) 3^{n}\left((-1)^{k} E_{k}+E_{k}\left(\frac{2}{3}\right)\right) \\
& \times\left((-1)^{n-k} E_{n-k}+E_{n-k}\left(\frac{2}{3}\right)\right) \frac{t^{n}}{n !} .
\end{aligned}
$$

By comparing the coefficient of both sides of $t^{n} / n$ ! and some calculations, we have the following equation:

$$
\frac{2}{3^{n}} \widetilde{U}_{n}(2)=\left(E_{n}\left(\frac{2}{3}\right)-E\right)^{n}+2^{n-1}\left(E_{n}\left(\frac{2}{3}\right)+(-1)^{n} E_{n}\right),
$$

with the usual convention of replacing $E^{n}$ by $E_{n}$.

Therefore, we consummated the proof of Theorem 8 .

From (26), we can suppose that

$$
\begin{aligned}
\widetilde{U}_{n}(x) & =\sum_{k=0}^{2 n} a(2 n, k) x^{k}+\sum_{k=1}^{2 n+1} b(2 n+1, k) x^{k} \\
& =\sum_{k=0}^{n} c(n, k) x^{k},
\end{aligned}
$$

where

$$
c(n, k)= \begin{cases}a(2 n, k) & \text { if } n=\text { odd }, \\ b(2 n+1, k) & \text { if } n=\text { even. }\end{cases}
$$

Therefore, we have Theorem 9.

Theorem 9. Let $n, v_{1}, \ldots, v_{k}, k \in \mathbb{N}$. Then one has

$$
\begin{aligned}
& k ! c(n, k) \\
& =\frac{3^{n-k} n !}{2^{k}} \\
& \times \sum_{\substack{v_{1}, \ldots, v_{k} \in \mathbb{N} \\
v_{1}+\cdots+v_{k}=n}}\left(\left(E_{v_{1}-1}\left(\frac{1}{3}\right)-E_{v_{1}-1}\left(\frac{2}{3}\right)+2 E_{v_{1}-1}\right)\right. \\
& \left.\quad \ldots\left(E_{v_{k}-1}\left(\frac{1}{3}\right)-E_{v_{k}-1}\left(\frac{2}{3}\right)+2 E_{v_{k}-1}\right)\right) \\
& \left.\times\left(v_{1} ! \cdots v_{k} !\right)^{-1}\right) .
\end{aligned}
$$

Proof. From Definition 1, we can differentiate the $k$-times as follows:

$$
\left.\sum_{n=k}^{\infty} \frac{d^{k}}{d x^{k}} \widetilde{U}_{n}(x)\right|_{x=0} \frac{t^{n}}{n !}=\left(\log \frac{e^{t}}{e^{t}+e^{-t}-1}\right)^{k} .
$$

Here we required that

$$
\begin{aligned}
& \log \frac{e^{t}}{e^{t}+e^{-t}-1} \\
& \quad=\sum_{n=0}^{\infty} \frac{3^{n}}{2}\left(E_{n}\left(\frac{1}{3}\right)-E_{n}\left(\frac{2}{3}\right)+2 E_{n}\right) \frac{t^{n+1}}{(n+1) !} \\
& \quad=\sum_{n=1}^{\infty} \frac{3^{n-1}}{2}\left(E_{n-1}\left(\frac{1}{3}\right)-E_{n-1}\left(\frac{2}{3}\right)+2 E_{n-1}\right) \frac{t^{n}}{n !} .
\end{aligned}
$$

Hence, we derive that

$$
\begin{gathered}
\left.\sum_{n=k}^{\infty} \frac{d^{k}}{d x^{k}} \widetilde{U}_{n}(x)\right|_{x=0} \frac{t^{n}}{n !} \\
\left.=\sum_{n=1}^{\infty} \frac{3^{n-1}}{2}\left(E_{n-1}\left(\frac{1}{3}\right)-E_{n-1}\left(\frac{2}{3}\right)+2 E_{n-1}\right) \frac{t^{n}}{n !}\right)^{k} \\
=\sum_{n=k}^{\infty} \frac{3^{n-k} n !}{2^{k}} \\
\quad \times \sum_{\substack{v_{1}, \ldots, v_{k} \in \mathbb{N} \\
v_{1}+\cdots+v_{k}=n}}\left(\left(\left(E_{v_{1}-1}\left(\frac{1}{3}\right)-E_{v_{1}-1}\left(\frac{2}{3}\right)+2 E_{v_{1}-1}\right)\right.\right. \\
\left.\quad \cdots\left(E_{v_{k}-1}\left(\frac{1}{3}\right)-E_{v_{k}-1}\left(\frac{2}{3}\right)+2 E_{v_{k}-1}\right)\right) \\
\left.\times\left(v_{1} ! \cdots v_{k} !\right)^{-1}\right) \frac{t^{n}}{n !} .
\end{gathered}
$$

By comparing the coefficient of both sides of $t^{n} / n$ !, we have the following equation:

$$
\begin{gathered}
\left.\frac{d^{k}}{d x^{k}} \widetilde{U}_{n}(x)\right|_{x=0} \\
=\frac{3^{n-k} n !}{2^{k}} \sum_{\substack{v_{1}, \ldots, v_{k} \in \mathbb{N} \\
v_{1}+\cdots+v_{k}=n}}\left(\left(\left(E_{v_{1}-1}\left(\frac{1}{3}\right)-E_{v_{1}-1}\left(\frac{2}{3}\right)+2 E_{v_{1}-1}\right)\right.\right. \\
\left.\cdots\left(E_{v_{k}-1}\left(\frac{1}{3}\right)-E_{v_{k}-1}\left(\frac{2}{3}\right)+2 E_{v_{k}-1}\right)\right) \\
\left.\times\left(v_{1} ! \cdots v_{k} !\right)^{-1}\right) .
\end{gathered}
$$

From (48), we easily see that

$$
\left.\frac{d^{k}}{d x^{k}} \widetilde{U}_{n}(x)\right|_{x=0}=k ! c(n, k) .
$$

Therefore, we clear off the proof of Theorem 9.

Theorem 10. Let $n, v_{1}, \ldots, v_{k}, k \in \mathbb{N}$. Then one has

$k ! c(n, k)$

$$
\begin{gathered}
=n ! \sum_{\substack{v_{1}, \ldots, v_{k} \in \mathbb{N} \\
v_{1}+\cdots+v_{k}=n}}\left(\left(\left((\widetilde{U}(x)-3)^{v_{1}-1}-(\widetilde{U}(x)-1)^{v_{1}-1}\right)\right.\right. \\
\left.\cdots\left((\widetilde{U}(x)-3)^{v_{k}-1}-(\widetilde{U}(x)-1)^{v_{k}-1}\right)\right) \\
\left.\times\left(v_{1} ! \cdots v_{k} !\right)^{-1}\right) .
\end{gathered}
$$

Proof. This proof can be proved by the similar method of Theorem 9. 
We also can derive relation polynomials $\widetilde{U}_{n}(x)$ and Euler numbers as follows.

Theorem 11. Let $n, k \in \mathbb{Z}^{+}$and $k+1 \leq n$. Then one derives

$$
\begin{aligned}
& \sum_{k=0}^{n-1}\left(\begin{array}{c}
n-1 \\
k
\end{array}\right)(-1)^{n-k-1}\left(2^{n-k}-1\right) \widetilde{U}_{k}(1) \\
& =\frac{3^{n-1}}{2}\left(E_{n-1}\left(\frac{1}{3}\right)-E_{n-1}\left(\frac{2}{3}\right)+2 E_{n-1}\right) .
\end{aligned}
$$

Proof. By the proof of Theorem 9, we can differentiate polynomials $\widetilde{U}_{n}(x)$ as follows:

$$
\begin{aligned}
\frac{d}{d t} & \left(\log \frac{e^{t}}{e^{t}+e^{-t}-1}\right) \\
& =\frac{2 e^{-t}-1}{e^{t}+e^{-t}-1} \\
& =\frac{e^{t}}{e^{t}+e^{-t}-1}\left(2 e^{-2 t}-e^{-t}\right) \\
& =\sum_{n=0}^{\infty} \widetilde{U}_{n}(1) \frac{t^{n}}{n !}\left(2 \sum_{n=0}^{\infty}(-2)^{n} \frac{t^{n}}{n !}-\sum_{n=0}^{\infty}(-1)^{n} \frac{t^{n}}{n !}\right) \\
& =\sum_{n=0}^{\infty} \sum_{k=0}^{n}\left(\begin{array}{l}
n \\
k
\end{array}\right)(-1)^{n-k}\left(2^{n-k+1}-1\right) \widetilde{U}_{k}(1) \frac{t^{n}}{n !} .
\end{aligned}
$$

By integrating from 0 to $t$, we deduce that

$$
\begin{aligned}
& \log \frac{e^{t}}{e^{t}+e^{-t}-1} \\
& \quad=\sum_{n=0}^{\infty} \sum_{k=0}^{n}\left(\begin{array}{l}
n \\
k
\end{array}\right)(-1)^{n-k}\left(2^{n-k+1}-1\right) \widetilde{U}_{k}(1) \frac{t^{n+1}}{(n+1) !} \\
& \quad=\sum_{n=1}^{\infty} \sum_{k=0}^{n-1}\left(\begin{array}{c}
n-1 \\
k
\end{array}\right)(-1)^{n-(k+1)}\left(2^{n-k}-1\right) \widetilde{U}_{k}(1) \frac{t^{n}}{n !} .
\end{aligned}
$$
that

We already knew from the proof process of Theorem 9

$$
\begin{aligned}
\log \frac{e^{t}}{e^{t}+e^{-t}-1} \\
\quad=\sum_{n=1}^{\infty} \frac{3^{n-1}}{2}\left(E_{n-1}\left(\frac{1}{3}\right)-E_{n-1}\left(\frac{2}{3}\right)+2 E_{n-1}\right) \frac{t^{n}}{n !} .
\end{aligned}
$$

By using the above equation, we can derive that

$$
\begin{aligned}
& \sum_{k=0}^{n-1}\left(\begin{array}{c}
n-1 \\
k
\end{array}\right)(-1)^{n-k-1}\left(2^{n-k}-1\right) \widetilde{U}_{k}(1) \\
& =\frac{3^{n-1}}{2}\left(E_{n-1}\left(\frac{1}{3}\right)-E_{n-1}\left(\frac{2}{3}\right)+2 E_{n-1}\right) .
\end{aligned}
$$

Therefore, we completely demonstrated the proof of Theorem 11.

\section{Acknowledgments}

The authors express their gratitude to the referee for his/her valuable comments. This work was supported by the National Research Foundation of Korea (NRF) grant funded by the Korean Government (NRF-2013-Fostering Core Leaders of the Future Basic Science Program).

\section{References}

[1] M. Açikgöz, D. Erdal, and S. Araci, "A new approach to $q$-Bernoulli numbers and $q$-Bernoulli polynomials related to $q$-Bernstein polynomials," Advances in Difference Equations, vol. 2010, Article ID 951764, 9 pages, 2010.

[2] A. Bayad, "Modular properties of elliptic Bernoulli and Euler functions," Advanced Studies in Contemporary Mathematics, vol. 20, no. 3, pp. 389-401, 2010.

[3] N. K. Govil and V. Gupta, "Convergence of $q$-Meyer-König-Zeller-Durrmeyer operators," Advanced Studies in Contemporary Mathematics, vol. 19, no. 1, pp. 97-108, 2009.

[4] M.-S. Kim and S. Hu, "On p-adic Hurwitz-type Euler zeta functions," Journal of Number Theory, vol. 132, no. 12, pp. 2977-3015, 2012.

[5] G. Liu, "The D numbers and the central factorial numbers," Publicationes Mathematicae Debrecen, vol. 79, no. 1-2, pp. 41-53, 2011.

[6] H. Ozden and Y. Simsek, "A new extension of $q$-Euler numbers and polynomials related to their interpolation functions," Applied Mathematics Letters, vol. 21, no. 9, pp. 934-939, 2008.

[7] S.-H. Rim, K. H. Park, and E. J. Moon, "On Genocchi numbers and polynomials," Abstract and Applied Analysis, vol. 2008, Article ID 898471, 7 pages, 2008.

[8] S.-H. Rim, J.-H. Jin, E.-J. Moon, and S.-J. Lee, "Some identities on the $q$-Genocchi polynomials of higher-order and $q$-Stirling numbers by the fermionic $p$-adic integral on $\mathbb{Z}_{p}$," International Journal of Mathematics and Mathematical Sciences, vol. 2010, Article ID 860280, 14 pages, 2010.

[9] C. S. Ryoo, "A numerical computation on the structure of the roots of q-extension of Genocchi polynomials," Applied Mathematics Letters, vol. 21, no. 4, pp. 348-354, 2008.

[10] Y. Simsek, V. Kurt, and D. Kim, "New approach to the complete sum of products of the twisted $(h, q)$-Bernoulli numbers and polynomials," Journal of Nonlinear Mathematical Physics, vol. 14, no. 1, pp. 44-56, 2007.

[11] Y. Simsek, "Generating functions of the twisted Bernoulli numbers and polynomials associated with their interpolation functions," Advanced Studies in Contemporary Mathematics, vol. 16, no. 2, pp. 251-278, 2008.

[12] Y. Simsek, “Twisted $(h, q)$-Bernoulli numbers and polynomials related to twisted $(h, q)$-zeta function and L-function," Journal of Mathematical Analysis and Applications, vol. 324, no. 2, pp. 790-804, 2006.

[13] Z.-H. Sun, "Congruences for sequences similar to Euler numbers," Journal of Number Theory, vol. 132, no. 4, pp. 675-700, 2012.

[14] H. M. Srivastava, B. Kurt, and Y. Simsek, "Some families of Genocchi type polynomials and their interpolation functions," Integral Transforms and Special Functions, vol. 23, no. 12, pp. 919938, 2012.

[15] Y. Simsek, "Generating functions for generalized Stirling type numbers, Array type polynomials, Eulerian type polynomials 
and their applications," Fixed Point Theory and Applications, vol. 2013, article 87, 2013.

[16] Y. Simsek, A. Bayad, and V. Lokesha, " $q$-Bernstein polynomials related to $q$-Frobenius-Euler polynomials, $l$-functions, and $q$ Stirling numbers," Mathematical Methods in the Applied Sciences, vol. 35, no. 8, pp. 877-884, 2012.

[17] R. Dere, Y. Simsek, and H. M. Srivastava, "A unified presentation of three families of generalized Apostol type polynomials based upon the theory of the umbral calculus and the umbral algebra," Journal of Number Theory, vol. 133, no. 10, pp. 3245-3263, 2013. 


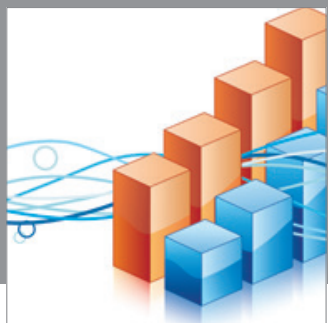

Advances in

Operations Research

mansans

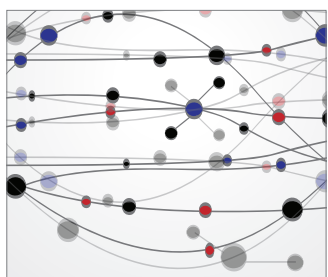

The Scientific World Journal
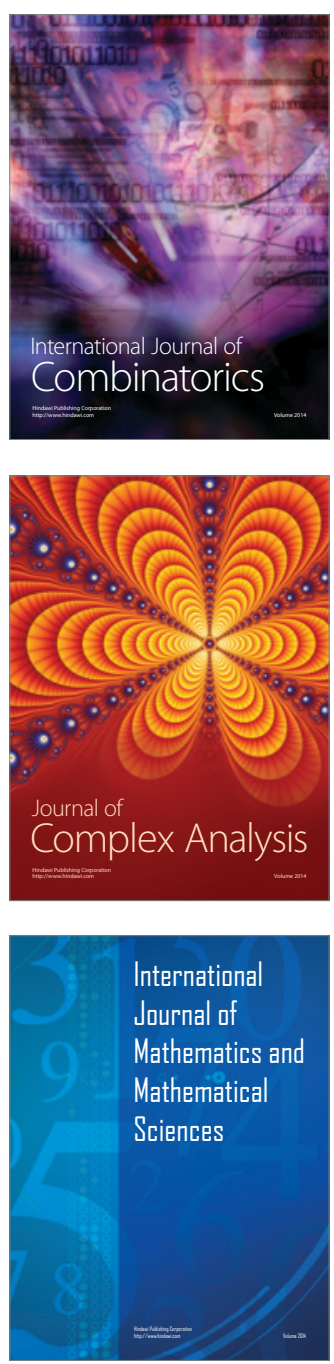
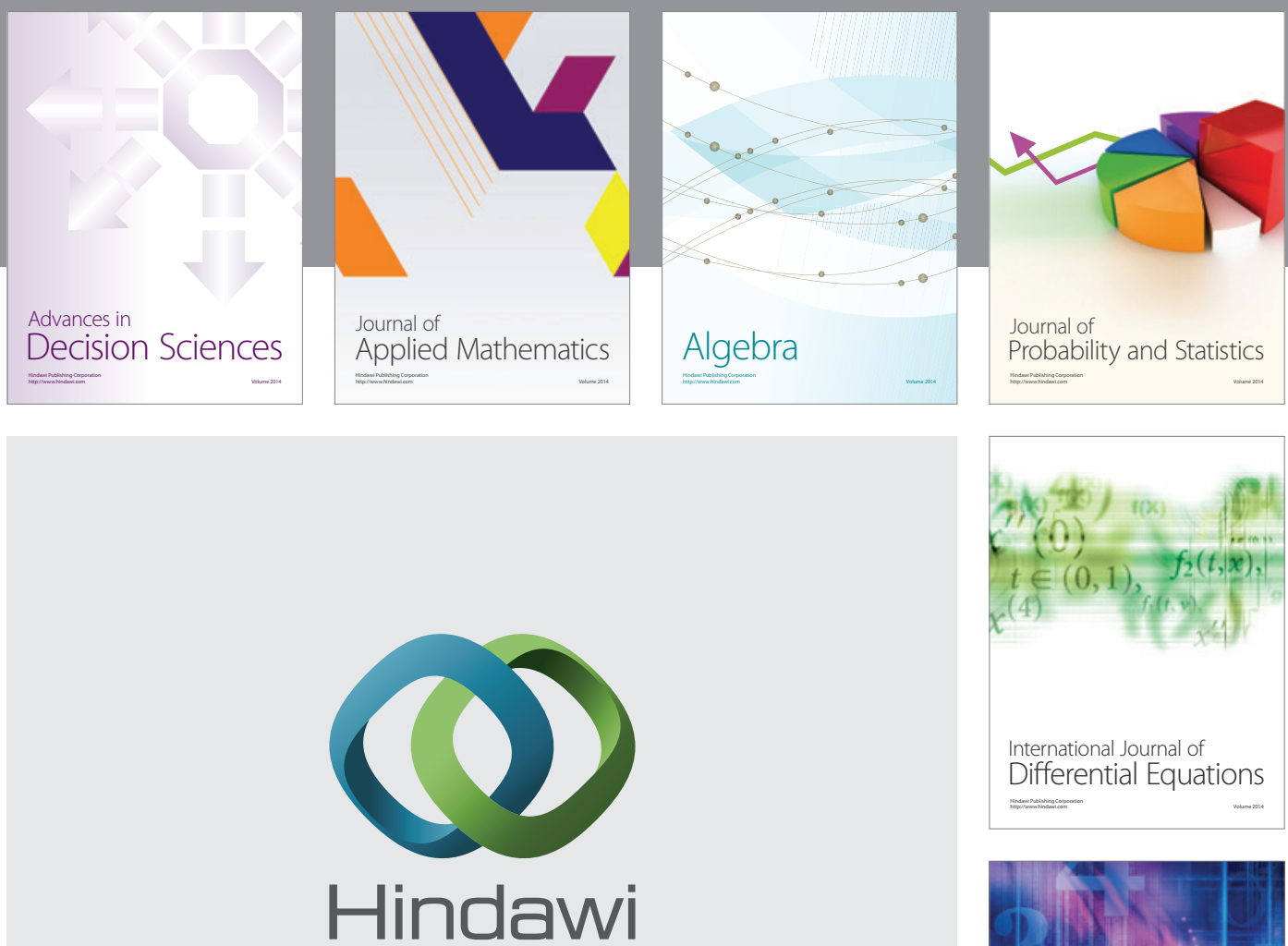

Submit your manuscripts at http://www.hindawi.com
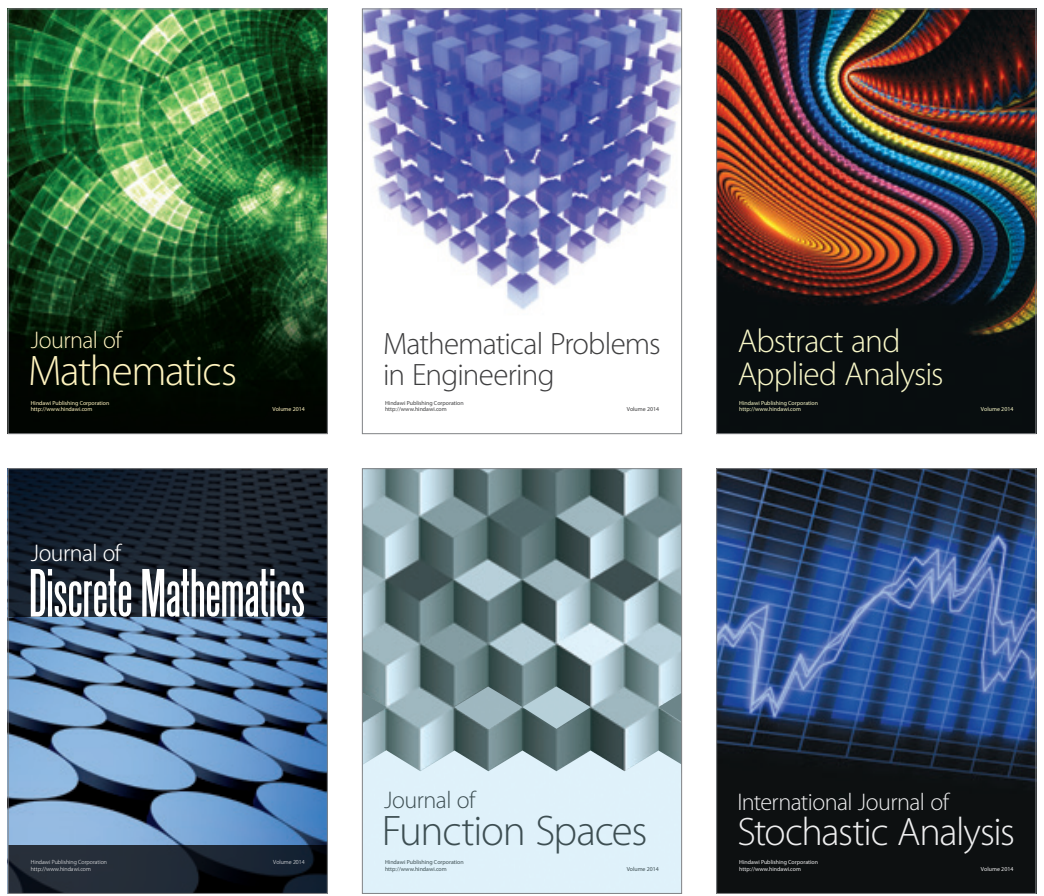

Journal of

Function Spaces

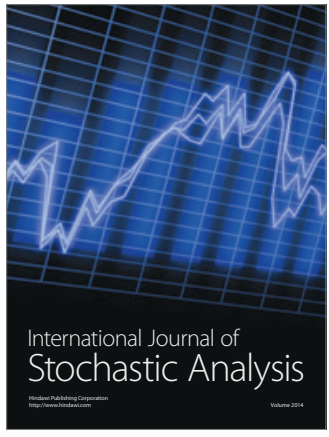

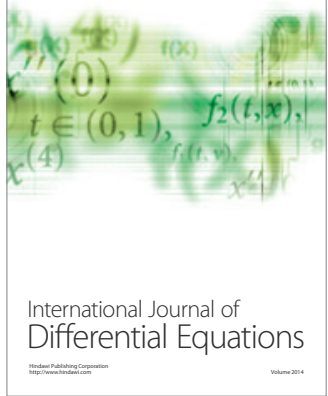
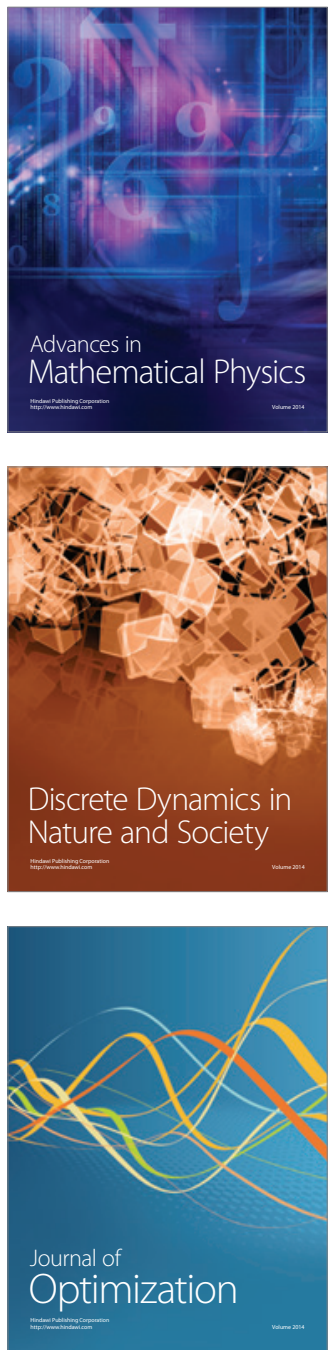